\title{
Unilateral pedal lymphangiography plus computed tomography angiography for location of persistent idiopathic chyle leakage not detectable by ordinary contrast computed tomography
}

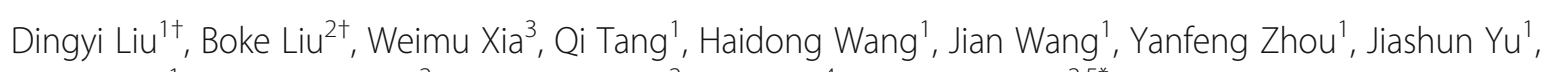
Wenmin $\mathrm{Li}^{1}$, Mingwei Wang ${ }^{2}$, Wenlong Zhou ${ }^{2}$, Sang $\mathrm{Hu}^{4}$ and Yuan Shao ${ }^{2,5^{*}}$

\begin{abstract}
Background: To identify the value of unilateral pedal lymphangiography (LPG) plus computed tomography angiography (CTA) in accurate depiction of persistent idiopathic chyluria undetectable by ordinary contrast CT.

Methods: Eighteen patients 44-63 years of age with persistent idiopathic chyluria who failed conservative management were included. Ordinary $C T$ had not revealed a chyle leak. Cystoscopy, unilateral LPG, and post-LPG CT angiography (CTA) were sequentially performed. Ligation and stripping of the perirenal lymphatics were subsequently performed guided by lymphangiography and CTA.

Results: LPG and post-LPG CTA detected 17 unilateral and one bilateral chyle leaks in the 18 patients, with clear images of the communication of lymphatic vessels and the renal collecting or vascular system. The success rate was significantly better than cystoscopy $(100 \%$ vs $50.0 \%, P=0.005)$ or $L P G$ alone $(100 \%$ vs. $72.2 \%, P=0.016)$. Chyluria resolved after surgery in all patients; no relapses were found.

Conclusions: LPG plus post-LPG CTA accurately characterized perirenal lymphangiectasia that was not demonstrated by routine contrast-enhanced $C T$ or not suitable for magnetic resonance imaging. Despite of its invasiveness, this method is a good diagnostic alternative to LPG in patients with persistent chyluria requiring surgery.
\end{abstract}

Keywords: Lymphangiography, Chyluria, Computerized tomographic angiography, Precise location

\section{Background}

Chyluria is the passage of chyle in the urine caused by the rupture of retroperitoneal lymphatics with leakage into the pyelocaliceal system, giving urine a milky appearance. The etiologies include thoracic duct stenosis, tuberculosis, cancer, trauma, pregnancy, filariasis,

\footnotetext{
* Correspondence: shaoyuan15@hotmail.com

${ }^{\dagger}$ Equal contributors

2Department of Urology, Shanghai Jiao Tong University Medical School Affiliated Ruijin Hospital, 197 Ruijin Er Road, Shanghai 200025, People's Republic of China

${ }^{5}$ Department of Urology, Shanghai Jiao Tong University Medical School Affiliated Ruijin Hospital North, 999 Xiwang Road, Shanghai 201801, People's Republic of China

Full list of author information is available at the end of the article
}

or the cause may not be clear. The result is dilatation of distal lymphatics and the eventual rupture of lymphatic vessels into the urinary collecting system [1, 2]. Although rare, severe fluid and protein loss may cause hypovolemia and hypoproteinemia in some patients. Cystoscopy, lymphangiography (LPG), computed tomography $(\mathrm{CT})$, magnetic resonance imaging (MRI) and lymphoscintigraphy are used to diagnose and locate the origin of chyluria [2-4]. Combining LPG with post-LPG CT imaging may increase the ability to locate chyle leaks $[2,5,6] .$, MRI is contraindicated in patients with implanted metal devices. Some patients fail conservative management because their chyle leaks are not visualized by routine contrast $\mathrm{CT}$, and require additional 
evaluation of the lymphatic system before treatment can be started. The aim of this study was to evaluate the value of unilateral LPG with post-LPG CT angiography (CTA) in chyluria patients who failed to conservative management with chyle leaks undetectable by ordinary contrast CT.

\section{Methods}

\section{Patients}

Eighteen patients diagnosed with persistent idiopathic chyluria between January 2013 and March 2017 were included. Ten were men and eight were women. Their median age was 51.5 (range 44-63) years, and the duration of chyluria ranged from 3 to 30 years. No patient had a history of tuberculosis or trauma. The main clinical manifestations were recurrent milky urine and asthenia. Seven patients experienced edema, one experienced severe anemia, 16 had intermittent recurrent chyluria, and two had persistent chyluria. All patients had test-confirmed chyluria; three had chylous hematuria. All were negative for filariasis antibody, urinalysis showed no urinary tract infections, other diseases cancer, and trauma were excluded. Conservative treatments such as bed rest, plenty of water with limited fat intake, and renal pelvic instillation via retrograde ureteral perfusion, had all failed in these patients. Renal pelvic sclerotherapy with povidone iodine or dextrose was performed one or two times in 11 patients without effect. The sites of chyle leaks could not be visualized with routine contrast CT. Cystoscopy including at least $5 \mathrm{~min}$ of observations of both ureteric orifices found unilateral urinary excretion of chyle in only nine patients (six left and three right). LPG CTA was subsequently performed.

\section{LPG}

The lymphatics were stained by injecting $2 \mathrm{ml}$ methylene blue into the web space between the first and second toes of one foot. A linear cut-down was performed on the dorsum of the foot below the ankle 30 min later to isolate a lymphatic vessel. After cannulation of the lymphatic vessel with a 30 gauge needle, iodized oil (Lipiodol; Laboratoire Guerbet, Roissy, France), a contrast agent for LPG, was injected at a rate of $0.1 \mathrm{ml} / \mathrm{min}$, not exceeding a total volume of $14 \mathrm{ml}$. CTA was performed immediately to assess apparent chyle leaks and to document the filling phase of the LPG.

\section{CTA}

CTA was performed with a $320 \times 0.5 \mathrm{~mm}$ detector row CT unit (Aquilion ONE, Toshiba, Japan). A $40 \mathrm{ml}$ volume of Iobitridol or Omnipaque nonionic contrast medium, $350 \mathrm{mg} / \mathrm{ml}$ was administered via an antecubital vein by bolus injection at rate $3-4 \mathrm{~mL} / \mathrm{s}$ using a power injector. Renal artery imaging scans were performed
20-30 s after injection, and scans of the urinary collecting system were performed after 3-5 min. Volume rendering (VR), maximum intensity projection (MIP) and multiplanar reformation (MPR) of the CT scans allowed accurate visualization of abdominal or retroperitoneal lymphatic vessels, lymphatic leakage, the kidney, renal artery, and the collecting system.

\section{Surgical treatment}

Renal lymphatic stripping and ligation were performed in all patients based on the anatomic location indicated by the LPG and post-LPG CTA. Surgical approach was decided according to the patient's condition, and both laparoscopy (4 cases) and open surgery (14 cases) were performed. A urine chyle test verified the therapeutic effect.

\section{Statistical analysis}

Qualitative variables were compared using the $\mathrm{X} 2$ test. $P$-values $<0.05$ considered statistically significant. The statistical analysis was performed with SPSS 20.0 (SPSS Inc., Chicago, IL, USA).

\section{Results}

LPG and post-LPG CTA succeeded in delineating the thoracic ducts and confirming the absence of obvious obstructions. Ten of the 18 patients had lympho-urinary fistulas on the left side, seven were on the right side, nine of which were consistent with the cystoscopy results. The remaining patient was diagnosed with unilateral chyluria by cystoscopy, LPG found bilateral lesions. LPG plus CTA was more successful than cystoscopy in locating the side of chyle leaks ( $100 \%$ vs. $50.0 \%, P=0.005$, Table 1$)$. When combined with post-LPG CTA, VR displayed the lymphatic reticular distribution in the kidney and renal fascia. In nine patients, VR and MIP revealed retroperitoneal lymphatic distortion, with reflux of lymphangiography contrast into the region of the contralateral iliac artery as well as showing the lymphatics adjacent to the renal arteries and veins (Fig. 1). LPG CTA provided detailed imaging information in all 18 patients. LPG alone provided imaging of equivalent value in only 13 patients. Accuracy of chyle leak location was better with LPG CTA than with LPG alone $(100 \%$ vs. $72.2 \%, P=0.016$; Table 2$)$. The

Table 1 The location of chyluria shown by cystoscopy and IPG $\subset T A$ Location Patient number Cystoscopy LPG+CTA LPG $p$-value* of chyluria

\begin{tabular}{clllll}
\hline Unilateral & 17 & $9(52.9 \%)$ & $17(100 \%)$ & 17 & 0.0004 \\
Left & 10 & 6 & $10(58.8 \%)$ & 10 & \\
Right & 7 & 3 & $7(41.2 \%)$ & 7 & \\
Bilateral & 1 & 0 & 1 & 1 & \\
Overall & 18 & $9(50.0 \%)$ & $18(100 \%)$ & 18 & 0.005
\end{tabular}

LPG lymphangiography, CTA computerized tomographic angiography ${ }^{*} \mathrm{X} 2$ test 


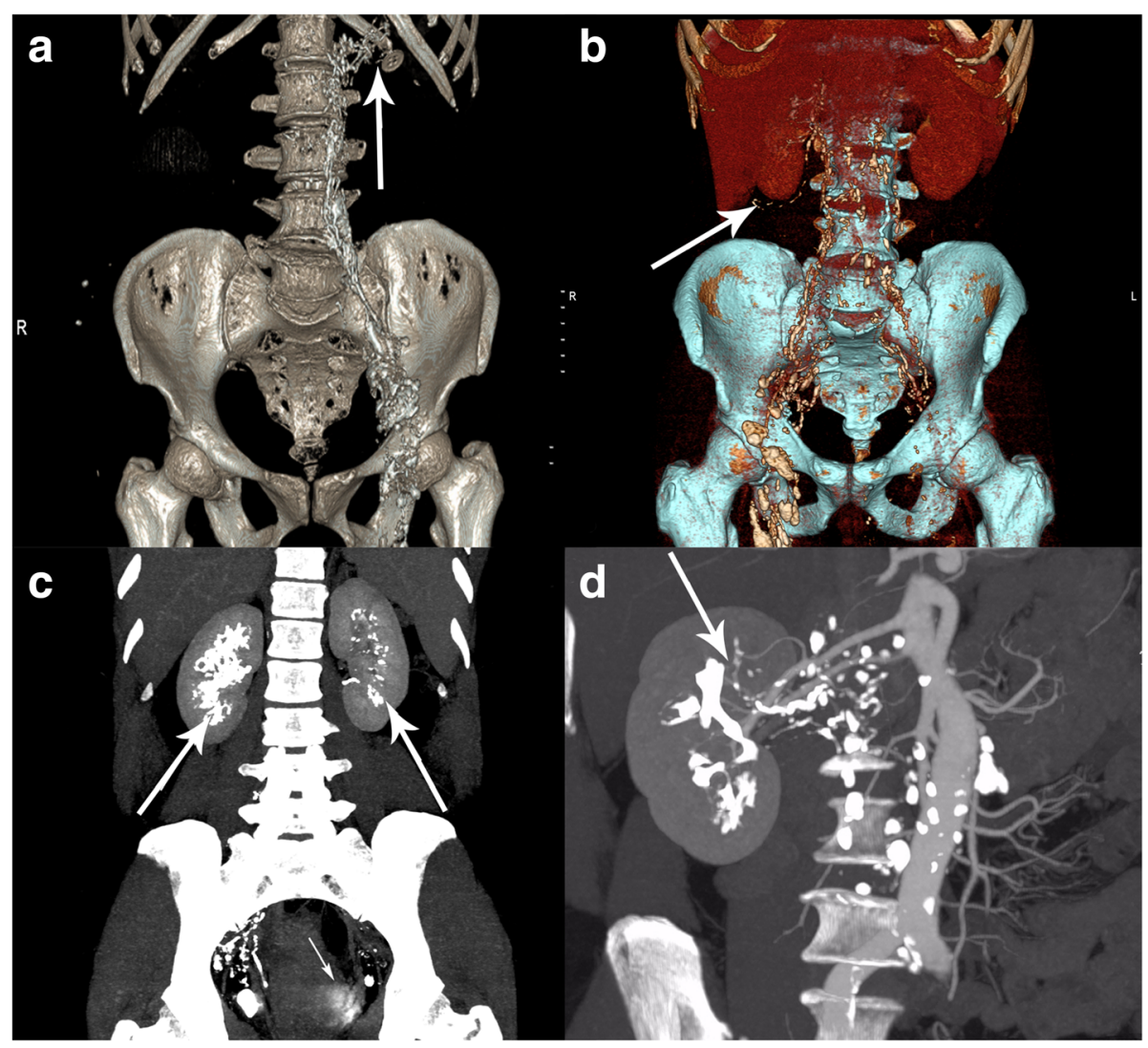

Fig. 1 VR of CT data showing (a) the distribution of lymphatic vessels around the left renal artery and vein. b Chyle leaks in right renal fascia. MIP of $\mathrm{CT}$ data showing (c) Bilateral lymphatic leakage of the renal pelvis, and reflux of contrast agent into the bladder and (d) right renal lymphatic leakage and lymphatic vessel lesions adjacent to the renal vein

results obtained with LPG CTA were sufficient to allow performing renal lymphatic stripping and ligation in all patients. Chyluria resolved immediately after surgery in 17 unilateral chyle leakage patients, with negative urine chyle tests. The remaining patient with bilateral lympho-urinary fistulas received renal lymphatic stripping on right side, which was the more severe side. His milky urine disappeared within 7 days after surgery, and a urine chyle test was negative. No recurrences were observed over a median follow-up 31 (range 8-52) months after surgery.

Table 2 Successful location of chyluria by cystoscopy, CT, LPG, and LPG CTA

\begin{tabular}{lllll}
\hline & Cystoscopy & $\mathrm{CT}$ & LPG & LPG + CTA \\
\hline Invasive & $\mathrm{Y}$ & $\mathrm{N}$ & $\mathrm{Y}$ & $\mathrm{Y}$ \\
Sides & $\mathrm{Y} / \mathrm{N}$ & $\mathrm{Y} / \mathrm{N}$ & $\mathrm{Y}$ & $\mathrm{Y}$ \\
Sites & $\mathrm{N}$ & $\mathrm{Y} / \mathrm{N}$ & $\mathrm{Y} / \mathrm{N}$ & $\mathrm{Y}$ \\
precise location & $\mathrm{N}$ & $\mathrm{N}$ & $13 / 18$ & $18 / 18^{*}$ \\
Radiation & $\mathrm{N}$ & $\mathrm{N}$ & $\mathrm{Y}$ & $\mathrm{Y}^{* *}$ \\
\hline
\end{tabular}

LPG lymphangiography, CTA computerized tomographic angiography, $Y$ Yes, $N$ No * $P=0.016$ (LPG CTA vs. LPG)

** more radiation exposure than LPG or CT alone

\section{Discussion}

Chyluria can be confirmed by a urine chyle test. About $80 \%$ of patients respond to conservative management with a low-fat, high-protein diet or intraperitoneal injection of sclerotherapy such as silver nitrate, povidone iodine or dextrose, about $22 \%$ relapse within 2 years [ 7 , 8]. Surgical intervention is needed for patients with severe chyluria patients who fail to respond to conservative management or have short-term relapses. Successful surgery requires clear identification and accurate location of the site of chyle leakage, especially the relationship between lymphatic vessels and the renal collection system or vascular system.

Imaging studies in patients with severe chyluria generally include cystoscopy and LPG. LPG is more successful than cystoscopy in detecting bilateral lymphatic renal pelvis fistulas than cystoscopy [2]. The appearance of LPG images in these chyluria patients was wire-like, with semicircular or coralline-shaped shadows that were primarily distributed in the renal pelvis and parenchyma (Fig. 2a). The renal hilus was distorted and dilated, lumbar or iliac lymph vessels could be seen, and obviously dilated truncus lumbalis 


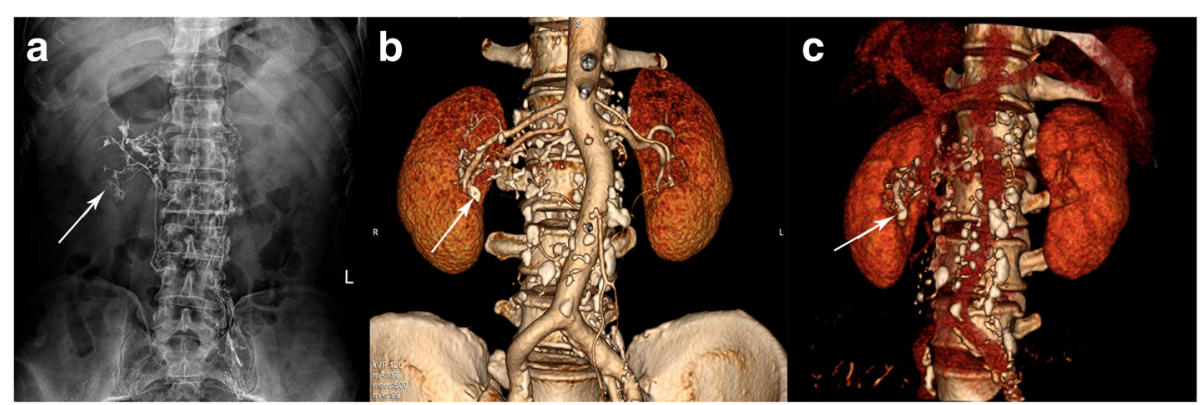

Fig. 2 A 60-year-old man with persistent idiopathic chyluria. a Pre-CTA LPG showing wire-like, semicircular shadow at the renal pelvis area and parenchyma in LPG (KUB) indicated right renal lymphatic leakage. b, $\mathbf{c} L P G$ CTA showing accumulation of contrast agent in right renal hilus

lymph vessels were occasionally observed. Single photon emission computed tomography (SPECT)/CT or MRI may be useful in the location of lymphatic ducts and chyle leakage sites [9-11], but LPG remains the most widely used method [12]. Evaluation of abdominal and retroperitoneal lymphatic abnormalities, including lymphatic leaks, using MRI lymphography with heavily T2-weighted fast spin echo sequences [10]. Nonenhanced MRI lymphangiography is a safe and effective method for imaging the central lymphatic system, and can contribute to differential diagnosis and appropriate preoperative evaluation of chylothorax or lymphangioma [13]. However there been few reports have described its use in chyluria patients [14].

We previously reported the successful use of LPG in diagnosing chyluria and LPG followed by a CT scan to directly show fistulae between the perinephric collection and lymphatic systems in either a plain scan or reconstructed image [2]. It is not clear whether a CT scan is of help after LPG. The CT increases the radiation exposure, and may not provide the information needed to perform the required surgery. It cannot show the details of the connections between lymphatic vessels and renal blood vessels, which may result in surgical failure because of incomplete ligation of all the lymphatic branches surrounding the renal arteries or veins. LPG combined with post-LPG CTA clearly show such structures and the relation of the lymphatic vessels and the renal collecting or vascular systems. In this study, the lymphatic lesions were well visualized by LPG with post-LPG CTA in all patients (Figs. 2 and 3), providing a reliable basis for renal pedicle lymphatic ligation and stripping.

Cystoscopy correctly found the side of the chyle leak in only about half the patients. LPG, the classic diagnostic tool [12], revealed not only the side of the lymphatic leaks, but also the approximate sites of reflux of contrast agent reflux into the renal collecting system (see Fig. 2a). However, LPG was unsatisfactory in some complicated cases, and it was difficult to obtain more information on the chyle leak in addition to the side of chyluria. LPG combined with post-LPG CTA clearly showed additional detail including the course of renal blood vessels, lymphatic vessels, the collection system, and their interlaced

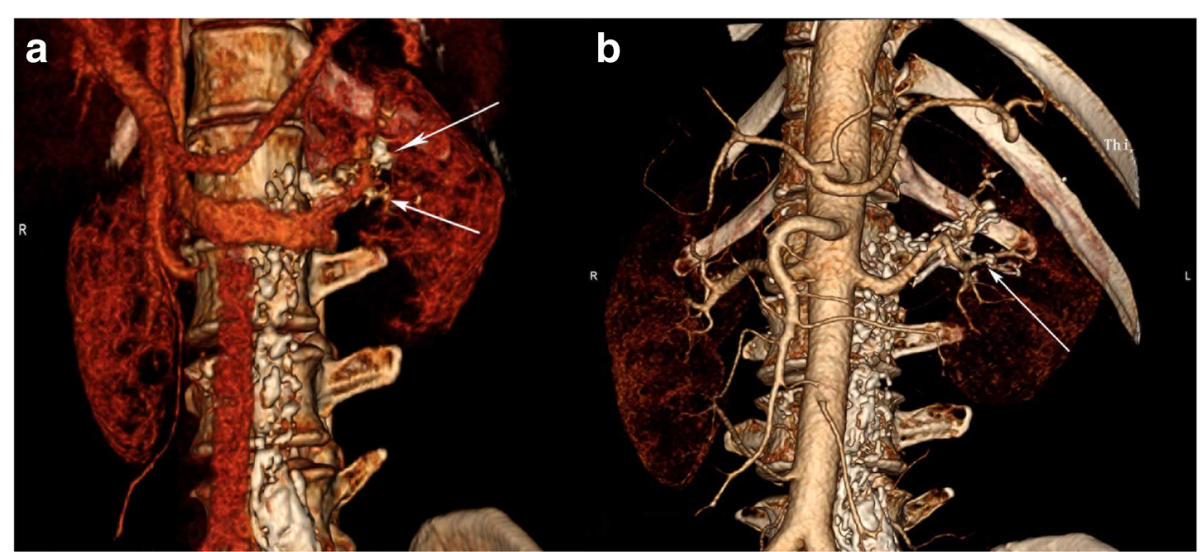

Fig. 3 A 61-year-old woman with persistent idiopathic chyluria. a, b LPG CTA showing contrast agent adjacent to the left renal artery and its branches into the kidney 
connections. The radiation exposure was more with LPG CTA than with LPG alone (Table 2), but in complicated cases in which CT or LPG alone were not satisfactory, LPG combined with post-LPG CTA provided precise location and clear imaging information, especially in cases not suitable for use of MRI. Despite its level of invasiveness, this method is a good option in the diagnosis of persistent chyluria requiring surgery. Fever and pain are the most frequent complications after LPG. Severe complications such as hemoptysis, wound infection, and embolism of blood vessels have been reported $[12,15,16]$, but did not occur in this patient series.

The study was limited by a small number of patients because of the rarity of persistent idiopathic chyluria and by the absence of a randomized control group. A multicenter, randomized control study with large number of patients is necessary to further investigate the advantages of LPG with CTA in the accurate location of chyle leaks and the management of chyluria.

\section{Conclusions}

LPG combine with post-LPG CTA could provide precise location and clear imaging information for chyluria patients which cannot be detected by routine contrast CT or not suitable for MR examination. Despite of its mild invasiveness, this method could be better than LPG alone and be a good option in the diagnosis of persistent chyluria requiring surgery.

\section{Abbreviations \\ CT: Computed tomography; CTA: Computerized tomographic angiography; LPG: Unilateral pedal lymphangiography; MIP: Maximum intensity projection; MPR: Multiplanar reformation; MRI: Magnetic resonance imaging; SPECT/ $C T$ : Single photon emission computed tomography/computed tomography; VR: Volume rendering}

\section{Acknowledgements}

The authors thank all the patients and their families for their cooperation during regular follow-up.

\section{Funding}

This article is supported by Major Subjects Project of Health and Med system, Pudong New District, Shanghai (PWZX2014-19); Science and Technology Innovation Project of Pudong New District, Shanghai (PKJ 2013-y33).

\section{Availability of data and materials}

The datasets used and analyzed in this study are available from the corresponding author on reasonable request.

\section{Authors' contributions}

$\mathrm{DL}, \mathrm{YS}$, and SH conceived and designed the study. DL, QT, WL, WZ, WX, and MW performed the surgery and the case follow-up. JW, YZ, and JY performed data acquisition. HW performed LPG and CTA, provided the figs. BL performed the data analysis and wrote the manuscript. DL and YS edited the manuscript. All authors reviewed the manuscript. All authors read and approved the final manuscript.

\section{Ethics approval and consent to participate}

All study procedures were performed following the ethical guidelines of the Declaration of Helsinki. This study was approved by the ethics committee of
Ruijin Hospital and Shanghai Punan Hospital. All study participants provided fully written informed consent.

\section{Consent for publication}

We have obtained written informed consent from the patient for publication of this article and all the accompanying images. A copy of the written consent is available for review by the journal editor.

\section{Competing interests}

The authors declare that they have no competing interests.

\section{Publisher's Note}

Springer Nature remains neutral with regard to jurisdictional claims in published maps and institutional affiliations.

\section{Author details}

'Department of Urology, Department of Radiology, Shanghai Punan Hospital, Shanghai, People's Republic of China. ${ }^{2}$ Department of Urology, Shanghai Jiao Tong University Medical School Affiliated Ruijin Hospital, 197 Ruijin Er Road, Shanghai 200025, People's Republic of China. ${ }^{3}$ Department of Urology,

Chinese People's Liberation Army Hospital 184, Yingtan, People's Republic of China. ${ }^{4}$ Department of Urology, Shanghai Post and Telecommunication Hospital, 666 Changle Road, Shanghai 200040, People's Republic of China. ${ }^{5}$ Department of Urology, Shanghai Jiao Tong University Medical School Affiliated Ruijin Hospital North, 999 Xiwang Road, Shanghai 201801, People's Republic of China.

Received: 30 October 2017 Accepted: 29 January 2018

Published online: 06 February 2018

\section{References}

1. Nandy PR, Dwivedi US, Vyas N, Prasad M, Dutta B, Singh PB. Povidone iodine and dextrose solution combination sclerotherapy in chyluria. Urology. 2004;64:1107-9. 1110

2. Liu DY, He HC, Zhou WL, et al. The advantages of unilateral pedal lymphography in the diagnosis of chyluria. Urol Int. 2015;94:215-9.

3. Kos S, Haueisen $H$, Lachmund U, Roeren T. Lymphangiography: forgotten tool or rising star in the diagnosis and therapy of postoperative lymphatic vessel leakage. Cardiovasc Intervent Radiol. 2007;30:968-73

4. Matsumoto T, Yamagami T, Kato T, et al. The effectiveness of lymphangiography as a treatment method for various chyle leakages. Br J Radiol. 2009:82:286-90.

5. Liu DY, Shao Y, Shi JX. Unilateral pedal lymphangiography with non-contrast computerized tomography is valuable in the location and treatment decision of idiopathic chylothorax. J Cardiothorac Surg. 2014;9:8

6. Yoshimatsu R, Yamagami T, Miura H, Matsumoto T. Prediction of therapeutic effectiveness according to $C T$ findings after therapeutic lymphangiography for lymphatic leakage. Jpn J Radiol. 2013;31:797-802.

7. Koga S, Nagata Y, Arakaki Y, Matsuoka M, Ohyama C. Unilateral pedal lymphography in patients with filarial chyluria. BJU Int. 2000;85:222-3.

8. Seleem MM, Eliwa AM, Elsayed ER, et al. Single versus multiple instillation of povidone iodine and urographin in the treatment of chyluria: a prospective randomised study. Arab J Urol. 2016:14:131-5.

9. Suh M, Cheon GJ, Seo HJ, Kim HH, Lee DS. Usefulness of additional SPECT/CT identifying Lymphatico-renal shunt in a patient with Chyluria. Nucl Med Mol Imaging. 2015;49:61-4.

10. Arrive $L$, Azizi $L$, Lewin $M$, et al. MR lymphography of abdominal and retroperitoneal lymphatic vessels. AJR Am J Roentgenol. 2007;189: 1051-8.

11. Zelmanovitz F. Location of regional intestinal lymphangiectasia using Tc-99m dextran lymphoscintigraphy. Clin Nucl Med. 1999;24:210-1.

12. Guermazi A, Brice P, Hennequin C, Sarfati E. Lymphography: an old technique retains its usefulness. Radiographics. 2003;23:1541-58. 1559-1560

13. Kim EY, Hwang HS, Lee HY, et al. Anatomic and functional evaluation of central Lymphatics with noninvasive magnetic resonance lymphangiography. Medicine (Baltimore). 2016;95:e3109.

14. El MS, Arrive L. Magnetic resonance lymphography of chyluria. Kidney Int. 2010;78:712. 
15. Deso S, Kabutey NK, Vilvendhan R, Kim D, Guermazi A.

Lymphangiography in the diagnosis, localization, and treatment of a lymphaticopelvic fistula causing chyluria: a case report. Vasc Endovasc Surg. 2010;44:710-3.

16. Kusumoto S, Imamura A, Watanabe K. Case report: the incidental lipid embolization to the brain and kidney after lymphography in a patient with malignant lymphoma: CT findings. Clin Radiol. 1991;44:279-80.

Submit your next manuscript to BioMed Central and we will help you at every step:

- We accept pre-submission inquiries

- Our selector tool helps you to find the most relevant journal

- We provide round the clock customer support

- Convenient online submission

- Thorough peer review

- Inclusion in PubMed and all major indexing services

- Maximum visibility for your research

Submit your manuscript at www.biomedcentral.com/submit 